PROCEEDINGS OF THE

AMERICAN MATHEMATICAL SOCIETY

Volume 140, Number 11, November 2012, Pages 3703-3713

S 0002-9939(2012)11211-0

Article electronically published on March 6, 2012

\title{
AN INDUCTIVE ANALYTIC CRITERION FOR FLATNESS
}

\author{
JANUSZ ADAMUS, EDWARD BIERSTONE, AND PIERRE D. MILMAN
}

(Communicated by Lev Borisov)

\begin{abstract}
We present a constructive criterion for flatness of a morphism of analytic spaces $\varphi: X \rightarrow Y$ (over $\mathbb{K}=\mathbb{R}$ or $\mathbb{C}$ ) or, more generally, for flatness over $\mathcal{O}_{Y}$ of a coherent sheaf of $\mathcal{O}_{X}$-modules $\mathcal{F}$. The criterion is a combination of a simple linear-algebra condition "in codimension zero" and a condition "in codimension one" which can be used together with the Weierstrass preparation theorem to inductively reduce the fibre-dimension of the morphism $\varphi$.
\end{abstract}

\section{INTRODUCTION}

The main result of this article is a constructive criterion for flatness of a morphism of analytic spaces $\varphi: X \rightarrow Y$ (over $\mathbb{K}=\mathbb{R}$ or $\mathbb{C}$ ) or, more generally, for flatness over $\mathcal{O}_{Y}$ of a coherent sheaf of $\mathcal{O}_{X}$-modules $\mathcal{F}$.

In the special case that $X=Y$ and $\varphi=\operatorname{id}_{X}$ (the identity morphism of $X$ ), our criterion reduces to the following "linear algebra criterion". In a neighbourhood of a point $a \in X$, an $\mathcal{O}_{X}$-module $\mathcal{F}$ can be presented as

$$
\mathcal{O}_{X}^{p} \stackrel{\Phi}{\longrightarrow} \mathcal{O}_{X}^{q} \longrightarrow \mathcal{F} \longrightarrow 0
$$

where $\Phi$ is given by multiplication by a $q \times p$-matrix of analytic functions. Let $r=\operatorname{rank} \Phi(a)$. Then $\mathcal{F}_{a}$ is $\mathcal{O}_{X, a}$-flat if and only if all minors of order $r+1$ of $\Phi$ vanish near $a$.

Our flatness criterion, in general, is a combination of a condition "in codimension zero" similar to the preceding and a condition "in codimension one" which can be used together with the Weierstrass preparation theorem to inductively reduce the fibre-dimension of the morphism $\varphi$.

To justify the criterion, we use it to give natural constructive proofs of several classical results - Hironaka's existence of the local flattener [7, Douady's openness of flatness [4, and Frisch's generic flatness theorem [5. The proofs are essentially a mix of linear algebra and appropriate applications of the Weierstrass preparation theorem.

For example, in the case $X=Y$, the linear algebra criterion above provides an immediate construction of the local flattener of $\mathcal{F}$ at $a$ (i.e., the largest germ of an analytic subspace $T$ of $X$ at $a$ such that $\mathcal{F}_{a}$ is $\mathcal{O}_{T}$-flat). We can simply

Received by the editors January 10, 2011 and, in revised form, April 25, 2011.

2010 Mathematics Subject Classification. Primary 13C11, 32B99; Secondary 14B25.

Key words and phrases. Flat, Weierstrass preparation, local flattener, generic flatness.

This research was partially supported by Natural Sciences and Engineering Research Council of Canada Discovery Grant OGP 355418-2008, Polish Ministry of Science Discovery Grant NN201 540538 (first author), and by NSERC Discovery Grants OGP 0009070 (second author) and OGP 0008949 (third author). 
take $\mathcal{O}_{T}=\mathcal{O}_{X} / \mathcal{I}$, where the ideal $\mathcal{I}$ is generated by the minors of order $r+1$ of $\Phi$. Hironaka's local flattener, in general, can be described using a similar linear algebra construction and the Weierstrass preparation theorem.

Algebraic formulation of the flatness criterion. Let $\varphi: Z \rightarrow W$ and $\lambda: T \rightarrow W$ denote morphisms of analytic space-germs, where $W$ is regular, and let $F$ denote a finite $\mathcal{O}_{Z}$-module. We are concerned with $\mathcal{O}_{T}$-flatness of the module $F \tilde{\otimes}_{\mathcal{O}_{W}} \mathcal{O}_{T}$, where $\tilde{\otimes}_{\mathcal{O}_{W}}$ denotes the analytic tensor product; i.e., the tensor product in the category of local analytic $\mathcal{O}_{W}$-algebras. (For a review of the analytic tensor product and its right-derived functor Tor, which is used below in the proof of Lemma [3.2. see [1, §2] or [6, §2].) Via the embedding $\left(\phi, \operatorname{id}_{Z}\right): Z \rightarrow W \times Z$ and the natural projection $\pi: W \times Z \rightarrow W$, we can view $F$ as an $\mathcal{O}_{W \times Z}$-module and therefore as an $\mathcal{O}_{W}$-module. Via an embedding $Z \hookrightarrow \mathbb{K}_{0}^{m}$ we can also replace $Z$ by $\mathbb{K}_{0}^{m}$ without changing the $\mathcal{O}_{W}$-module structure of $F$. In particular, then $\mathcal{O}_{Z}=\mathbb{K}\{x\}=\mathbb{K}\left\{x_{1}, \ldots, x_{m}\right\}, \mathcal{O}_{T}=R / J$ for an appropriate ideal $J$ in $R:=\mathbb{K}\{y\}=\mathbb{K}\left\{y_{1}, \ldots, y_{n}\right\}$, and $\mathcal{O}_{W \times Z}=R\{x\}:=\mathbb{K}\left\{y, x_{1}, \ldots, x_{m}\right\}$. Let $A:=\mathcal{O}_{W \times Z}$. Let $\mathfrak{m}$ denote the maximal ideal $\left(y_{1}, \ldots, y_{n}\right)$ of $R$, and let $\mathfrak{n}=\mathfrak{m}+\left(x_{1}, \ldots, x_{m}\right) \subset A$. Then $\mathfrak{n}$ is the maximal ideal of $A$. Given a power series $f=f(y, x) \in A$, we denote by $f(0)$ or by $f(0, x)$ its evaluation at $y=0$, i.e., the image of $f$ under the homomorphism $A \rightarrow A(0):=A \tilde{\otimes}_{R} R / \mathfrak{m}$ of $R$-modules. Similarly, given an $A$-submodule $M$ of $A^{q}$, we denote by $M(0)$ the evaluation of $M$ at $y=0$, i.e., $M(0)=\left\{m(0) \in A(0)^{q}: m \in M\right\}$. In particular, $A(0) \cong \mathbb{K}\{x\}$.

We are thus interested in flatness of $F \tilde{\otimes}_{R} R / J$ over $R / J$, where $F$ is a finitely generated $A$-module and $J$ is an ideal in $R$.

Theorem 1.1. Let $R, A, F$ and $J$ be as above. Then:

(A) There exist $g \in A, l \in \mathbb{N}$ and a homomorphism $\psi: A^{l} \rightarrow F$ of A-modules such that $g(0, x) \neq 0, g \cdot F \subset \operatorname{im} \psi$ and $\operatorname{ker} \psi \subset \mathfrak{m} \cdot A^{l}$.

(B) $F \tilde{\otimes}_{R} R / J$ is a flat $R / J$-module if and only if, for any $g, l$ and $\psi$ as in (A), the following two conditions hold:

(1) $\operatorname{ker} \psi \subset J \cdot A^{l}$;

(2) $(F / \operatorname{im} \psi) \tilde{\otimes}_{R} R / J$ is a flat $R / J$-module.

Remark 1.2. The above theorem allows one to study flatness of a module $F$ by repeated reduction of the fibre-dimension over $R$. Indeed, consider $g$ and $\psi$ as in (A). First suppose that $g(0,0)=0$. Since $g(0, x) \neq 0$, we can apply the Weierstrass division theorem (after a generic linear coordinate change in $x$ ) to conclude that $A /(g \cdot A)$ is a finite $R\{\tilde{x}\}$-module, where $\tilde{x}=\left(x_{1}, \ldots, x_{m-1}\right)$. Then $F / \operatorname{im} \psi$ is a finite $R\{\tilde{x}\}$-module too, since $g \cdot F \subset \operatorname{im} \psi$. On the other hand, if $g(0,0) \neq 0$ (which is the case when the number of $x$-variables is 0 ), then condition (2) of (B) in the theorem is vacuous and no fibre dimension reduction is needed.

Proof of Theorem 1.1(A). Consider a presentation of $F$ as an $A$-module

$$
A^{p} \stackrel{\Phi}{\longrightarrow} A^{q} \stackrel{\Psi}{\longrightarrow} F \rightarrow 0 .
$$

By applying $\tilde{\otimes}_{R} R / J$ and $\tilde{\otimes}_{R} R / \mathfrak{m}$ to (1.1), we get presentations

$$
A^{p} / J \cdot A^{p} \stackrel{\Phi_{J}}{\longrightarrow} A^{q} / J \cdot A^{q} \stackrel{\Psi_{J}}{\longrightarrow} F \tilde{\otimes}_{R} R / J \rightarrow 0
$$

and

$$
A^{p} / \mathfrak{m} \cdot A^{p} \stackrel{\Phi_{\mathfrak{m}}}{\longrightarrow} A^{q} / \mathfrak{m} \cdot A^{q} \stackrel{\Psi_{\mathfrak{m}}}{\longrightarrow} F \tilde{\otimes}_{R} R / \mathfrak{m} \rightarrow 0
$$


of $F \tilde{\otimes}_{R} R / J$ and $F \tilde{\otimes}_{R} R / \mathfrak{m}$ respectively. Notice that identifying $\Phi$ with a matrix (with entries in $A$ ), $\Phi_{\mathfrak{m}}$ becomes the matrix with entries obtained by evaluating the corresponding entries of $\Phi$ at $y=0$.

Let $r_{\mathfrak{m}}:=\operatorname{rank}\left(\Phi_{\mathfrak{m}}\right)$. Choose an ordering of the columns and rows of $\Phi$ so that $\Phi$ can be written in block form as

$$
\Phi=\left[\begin{array}{ll}
\alpha & \beta \\
\gamma & \delta
\end{array}\right]
$$

where the matrix $\alpha$ is of size $r_{\mathfrak{m}} \times r_{\mathfrak{m}}$ and $(\operatorname{det} \alpha)(0)=(\operatorname{det} \alpha)(0, x) \neq 0$ in $A(0)$.

Let $\alpha^{\#}$ denote the adjoint matrix of $\alpha$, i.e., an $r_{\mathfrak{m}} \times r_{\mathfrak{m}}$ matrix with $\alpha^{\#} \cdot \alpha=$ $\alpha \cdot \alpha^{\#}=(\operatorname{det} \alpha) \cdot \operatorname{Id}_{r_{\mathrm{m}}}$.

Now, take $g:=\operatorname{det} \alpha, l:=q-r_{\mathfrak{m}}$, and let $\psi$ be the restriction of $\Psi: A^{r_{\mathfrak{m}}} \oplus A^{l} \rightarrow F$ to $\{0\}^{r_{\mathfrak{m}}} \oplus A^{l} \cong A^{l}$. Then $g(0, x) \neq 0$. The condition $g \cdot F \subset \operatorname{im} \psi$ is equivalent to saying that, for every vector $(\varrho, \sigma) \in A^{r_{\mathrm{m}}} \oplus A^{l}$, there exists $\sigma^{\prime} \in A^{l}$ such that $\Psi(g \cdot(\varrho, \sigma))=\Psi\left(\left(0, \sigma^{\prime}\right)\right)$ or, equivalently, that $g \cdot A^{q} \subset \operatorname{ker} \Psi+\left(\{0\}^{r_{\mathfrak{m}}} \oplus A^{l}\right)=$ $\operatorname{im} \Phi+\left(\{0\}^{r_{\mathfrak{m}}} \oplus A^{l}\right)$. But the latter follows from the fact that $g \cdot A^{r_{\mathfrak{m}}} \subset \operatorname{im} \alpha$.

Finally, by the choice of $\psi, \sigma \in \operatorname{ker} \psi$ if and only if $(0, \sigma) \in \operatorname{im} \Phi \cap\left(\{0\}^{r_{\mathfrak{m}}} \oplus A^{l}\right)$. Then $(0, \sigma)=\Phi((\xi, \eta))$ for some $(\xi, \eta) \in A^{r_{\mathfrak{m}}} \oplus A^{p-r_{\mathfrak{m}}}$ with $\alpha \xi+\beta \eta=0$. By the choice of $r_{\mathfrak{m}}$, every row of $[\gamma, \delta]$ is an $A(0)$-linear combination of the rows of $[\alpha, \beta]$ modulo $\mathfrak{m}$. Hence $\alpha \xi+\beta \eta=0$ implies that $\gamma \xi+\delta \eta \in \mathfrak{m} \cdot A^{l}$, i.e., that $\sigma \in \mathfrak{m} \cdot A^{l}$.

Theorem 1.1(B) is the main result of this article. We will prove it in Section 3.

Remark 1.3. Throughout the paper we will use the fact that the entries of the matrix $g \cdot \delta-\gamma \cdot \alpha^{\#} \cdot \beta$ are the $\left(r_{\mathfrak{m}}+1\right) \times\left(r_{\mathfrak{m}}+1\right)$ minors of $\Phi$. This is an immediate consequence of the following matrix identity: For any $q \times p$ block matrix (1.4), where $\alpha$ is of size $r \times r$,

$$
g \cdot \Phi=\left[\begin{array}{cc}
\alpha & 0 \\
\gamma & \operatorname{Id}_{q-r}
\end{array}\right] \cdot\left[\begin{array}{cc}
g \cdot \operatorname{Id}_{r} & \alpha^{\#} \cdot \beta \\
0 & g \cdot \delta-\gamma \cdot \alpha^{\#} \cdot \beta
\end{array}\right],
$$

where $g=\operatorname{det} \alpha$.

\section{Applications: LOCAl flattener, openness of flatness, GENERIC FLATNESS}

Theorem 2.1 (Hironaka's local flattener [7). Let $\varphi: Z \rightarrow W$ be a morphism of analytic space-germs, where $W$ is regular. Let $F$ be a finite $\mathcal{O}_{Z}$-module. Then there exists a unique analytic subgerm $P$ of $W$ (i.e., a unique local analytic $\mathbb{K}$-algebra $\mathcal{O}_{P}$ which is a quotient of $\left.\mathcal{O}_{W}\right)$ such that:

(1) $F \tilde{\otimes}_{\mathcal{O}_{W}} \mathcal{O}_{P}$ is $\mathcal{O}_{P}$-flat.

(2) Let $\lambda_{P}: P \rightarrow W$ denote the embedding. Then, for every morphism $\lambda: T \rightarrow$ $W$ of germs of analytic spaces such that $F \tilde{\otimes}_{\mathcal{O}_{W}} \mathcal{O}_{T}$ is $\mathcal{O}_{T}$-flat, there exists a unique morphism $\mu: T \rightarrow P$ such that $\lambda=\lambda_{P} \circ \mu$.

Remark 2.2. Suppose that $\lambda: T \rightarrow W$ is a morphism such that $F \tilde{\otimes}_{\mathcal{O}_{W}} \mathcal{O}_{T}$ is $\mathcal{O}_{T}$-flat. Since flatness is preserved by base change (see [7, Prop.6.8]), it follows that $\left(F \tilde{\otimes}_{\mathcal{O}_{W}} \mathcal{O}_{T}\right) \tilde{\otimes}_{\mathcal{O}_{T}} S$ is $S$-flat, for every subring $S$ of $\mathcal{O}_{T}$. In particular, identifying $\mathcal{O}_{W} / \operatorname{ker} \lambda^{*}$ with $\operatorname{im} \lambda^{*}$, we get that $F \tilde{\otimes}_{\mathcal{O}_{W}}\left(\mathcal{O}_{W} / \operatorname{ker} \lambda^{*}\right) \cong\left(F \tilde{\otimes}_{\mathcal{O}_{W}} \mathcal{O}_{T}\right)$ $\tilde{\otimes}_{\mathcal{O}_{T}}\left(\mathcal{O}_{W} / \operatorname{ker} \lambda^{*}\right)$ is $\left(\mathcal{O}_{W} / \operatorname{ker} \lambda^{*}\right)$-flat. Therefore, in Theorem 2.1 it suffices to consider an embedding $\lambda: T \rightarrow W$ and to show that there is an ideal $I(F)$ in $\mathcal{O}_{W}$ such that $F \tilde{\otimes}_{\mathcal{O}_{W}}\left(\mathcal{O}_{W} / J\right)$ is $\mathcal{O}_{W} / J$-flat if and only if $I(F) \subset J$. 
The germ $P$ is called the local flattener of $F$ (with respect to $\varphi$ ), and $I(F)$ is the ideal of the local flattener.

Proof of Theorem 2.1. The uniqueness of $P$ is automatic, since $\lambda_{P}^{*}: \mathcal{O}_{W} \rightarrow \mathcal{O}_{P}$ is surjective.

By regularity of $W$, we can identify $\mathcal{O}_{W}$ with the ring $R=\mathbb{K}\{y\}$ of convergent power series in $y=\left(y_{1}, \ldots, y_{n}\right)$. Assume that $Z$ is a subgerm of $\mathbb{K}_{0}^{m}$. Using the graph of $\varphi$ to embed $Z$ in $W \times \mathbb{K}^{m}$, we can think of $\mathcal{O}_{Z}$ as a quotient ring of $A=R\{x\}$, where $x=\left(x_{1}, \ldots, x_{m}\right)$. Then $F$ is a finitely generated $A$-module. We will proceed by induction on $m$, the number of the $x$-variables.

Choose $g \in A$ and $\psi: A^{l} \rightarrow F$ satisfying Theorem 1.1(A). Let $J(F)$ be the ideal in $R$ generated by the coefficients of (the expansions in $x$ of) the elements in ker $\psi$, i.e., the unique minimal ideal $J$ in $R$ satisfying $\operatorname{ker} \psi \subset J$. $A^{l}$. If $F=\operatorname{im} \psi$ (which is the case if $m=0$, since then $g$ is invertible in $A$ ), then Theorem 1.1(B) implies that $J(F)$ is the ideal of the local flattener of $F$. If $F \neq \operatorname{im} \psi$, then $m>0$ and we may assume by the inductive hypothesis (see Remark 1.2) that there is a local flattening ideal $I(F / \operatorname{im} \psi)$ in $\mathcal{O}_{W}$. It follows that $I(F):=J(F)+I(F / \operatorname{im} \psi)$ is the ideal of the local flattener of $F$.

Let $X$ and $Y$ be analytic spaces over $\mathbb{K}$, and let $\varphi: Y \times X \rightarrow Y$ be the canonical projection. Let $\mathcal{F}$ be a coherent $\mathcal{O}_{Y \times X}$-module. For $(\eta, \xi) \in Y \times X$, let $I_{\eta, \xi}(\mathcal{F})$ denote the ideal in $\mathcal{O}_{Y, \eta}$ of the local flattener of the stalk $\mathcal{F}_{(\eta, \xi)}$ (with respect to $\varphi$ ). Given any ideal $J$ in $\mathcal{O}_{Y, \eta}$, we let $J_{\eta^{\prime}}$ denote the ideal generated by (a system of generators of) $J$ at nearby points $\eta^{\prime} \in Y$. Then Theorem 1.1 implies the following.

Theorem 2.3 (Openness of flatness). For every $(\eta, \xi)$ in a sufficiently small open neighbourhood of $\left(\eta_{0}, \xi_{0}\right)$ in $Y \times X$, with $\eta$ in a representative of the zero-set germ $\mathcal{V}\left(I_{\eta_{0}, \xi_{0}}(\mathcal{F})\right)$, we have

$$
I_{\eta, \xi}(\mathcal{F}) \subset\left(I_{\eta_{0}, \xi_{0}}(\mathcal{F})\right)_{\eta}
$$

Remark 2.4 (Douady's openness of flatness 44). Let $\varphi: X \rightarrow Y$ be a morphism of analytic spaces, and let $\mathcal{F}$ be a coherent sheaf of $\mathcal{O}_{X}$-modules. Let $J$ be a coherent sheaf of ideals in $\mathcal{O}_{Y}$, and let $Z$ be the closed analytic subspace of $Y$ defined by $J$ (i.e., $\mathcal{O}_{Z}=\mathcal{O}_{Y} / J$ and $\left.|Z|=\operatorname{supp}\left(\mathcal{O}_{Y} / J\right)\right)$. Then Theorem 2.3 implies that

$$
N_{X}(Z)=\left\{\xi \in \varphi^{-1}(|Z|): \mathcal{F}_{\xi} \tilde{\otimes}_{\mathcal{O}_{Y, \varphi(\xi)}} \mathcal{O}_{Z, \varphi(\xi)} \text { is not } \mathcal{O}_{Z, \varphi(\xi)} \text {-flat }\right\}
$$

is a closed subset of $|X|$. In particular, for $Z=Y$, the latter implies openness of the set of points $\xi \in X$ with the property that $\mathcal{F}_{\xi}$ is a flat $\mathcal{O}_{Y, \varphi(\xi)}$-module. This result is due to Douady [4] and is the classical form of "openness of flatness".

Proof of Theorem 2.3. As in the proof of Theorem 2.1, we proceed by induction on the fibre-dimension $m$ of $\varphi: X \times Y \rightarrow Y$. Using Theorem 1.1(A) with $F=\mathcal{F}_{\left(\eta_{0}, \xi_{0}\right)}$, we can choose neighbourhoods $U$ of $\xi_{0}$ and $V$ of $\eta_{0}$, a function $g$ analytic on $V \times U$, and a morphism $\psi:\left.\mathcal{O}_{V \times U}^{l} \rightarrow \mathcal{F}\right|_{V \times U}$ of $\mathcal{O}_{V \times U}$-modules, such that $g\left(\eta_{0}, x\right) \neq 0$, $g_{\left(\eta_{0}, \xi_{0}\right)} \cdot \mathcal{F}_{\left(\eta_{0}, \xi_{0}\right)} \subset(\operatorname{im} \psi)_{\left(\eta_{0}, \xi_{0}\right)}$ and $(\operatorname{ker} \psi)_{\left(\eta_{0}, \xi_{0}\right)} \subset \mathfrak{m}_{V, \eta_{0}} \cdot \mathcal{O}_{V \times U,\left(\eta_{0}, \xi_{0}\right)}^{l}$. Since our problem is local, we can assume that $U$ (resp. $V$ ) is an open polydisc in $\mathbb{C}^{m}$ (resp. $\left.\mathbb{C}^{n}\right)$ centred at $\xi_{0}$ (resp. $\eta_{0}$ ). (After shrinking $V$ if necessary) let $J$ be a coherent $\mathcal{O}_{V^{-}}$-ideal such that $J_{\eta_{0}}=I_{\eta_{0}, \xi_{0}}(\mathcal{F})$; we can assume that $J_{\eta}=\left(I_{\eta_{0}, \xi_{0}}(\mathcal{F})\right)_{\eta}$ for all $\eta \in V$. Let $Z$ denote the closed analytic subspace of $V$ defined by $J$; i.e., $|Z|$ is a representative in $V$ of the zero-set germ $\mathcal{V}\left(I_{\eta_{0}, \xi_{0}}(\mathcal{F})\right)$. Then Theorem 1.1(B) 
implies that

$$
\begin{gathered}
(\operatorname{ker} \psi)_{\left(\eta_{0}, \xi_{0}\right)} \subset J_{\eta_{0}} \cdot \mathcal{O}_{V \times U,\left(\eta_{0}, \xi_{0}\right)}^{l}, \\
(\mathcal{F} / \operatorname{im} \psi)_{\left(\eta_{0}, \xi_{0}\right)} \tilde{\otimes}_{\mathcal{O}_{Y, \eta_{0}}} \mathcal{O}_{Z, \eta_{0}} \text { is } \mathcal{O}_{Z, \eta_{0}} \text {-flat. }
\end{gathered}
$$

It follows (after shrinking $U$ and $V$ if needed) that $g(\eta, x) \neq 0$ for all $\eta \in V$ and $g \cdot \mathcal{F} \subset \operatorname{im} \psi$. Then (2.2) implies that

$$
(\operatorname{ker} \psi)_{(\eta, \xi)} \subset J_{\eta} \cdot \mathcal{O}_{V \times U,(\eta, \xi)}^{l} \subset \mathfrak{m}_{V, \eta} \cdot \mathcal{O}_{V \times U,(\eta, \xi)}^{l},
$$

for all $(\eta, \xi) \in V \times U$ with $\eta \in|Z|$.

If $g(\eta, \xi) \neq 0$ (which is the case if $m=0$ ), then, by Theorem 1.1(B), the first inclusion of (2.4) implies that $I_{\eta, \xi}(\mathcal{F}) \subset J_{\eta}=\left(I_{\eta_{0}, \xi_{0}}(\mathcal{F})\right)_{\eta}$, as required.

Otherwise $g\left(\eta_{0}, \xi_{0}\right)=0$ (and $m>0$ ). By Theorem 1.1, it suffices to show that $(\mathcal{F} / \operatorname{im} \psi)_{(\eta, \xi)} \tilde{\otimes}_{\mathcal{O}_{Y, \eta}} \mathcal{O}_{Z, \eta}$ is $\mathcal{O}_{Z, \eta}$-flat, provided $\eta \in|Z|$ and $g(\eta, \xi)=0$. After a linear change of the $x$-variables, we can assume that $U=U^{\prime} \times U^{\prime \prime}$, where $U^{\prime}$ is spanned by the variables $\tilde{x}=\left(x_{1}, \ldots, x_{m-1}\right)$ and $U^{\prime \prime}$ is spanned by $x_{m}$, and that $g_{\left(\eta_{0}, \xi_{0}\right)}$ is regular in $x_{m}-\xi_{0 m}$, where $\xi_{0 m}$ is the last coordinate of $\xi_{0}$. By Remark [1.2, after shrinking $U$ if needed, we can consider $\mathcal{F} / \operatorname{im} \psi$ as a coherent $\mathcal{O}_{V \times U^{\prime}}$-module; we denote it $\tilde{\mathcal{F}}$. Let $\tilde{\xi}_{0}$ denote the $\tilde{x}$-coordinates of $\xi_{0}$. Then $\tilde{\mathcal{F}}_{\left(\eta_{0}, \tilde{\xi}_{0}\right)}=(\mathcal{F} / \operatorname{im} \psi)_{\left(\eta_{0}, \xi_{0}\right)}$ (since $g\left(\eta_{0}, \tilde{\xi}_{0}, \cdot\right)$ vanishes only at $\left.\xi_{0 m}\right)$, and hence $\tilde{\mathcal{F}}_{\left(\eta_{0}, \tilde{\xi}_{0}\right)} \tilde{\otimes}_{\mathcal{O}_{Y, \eta_{0}}} \mathcal{O}_{Z, \eta_{0}}$ is $\mathcal{O}_{Z, \eta_{0}}$ flat, by (2.3). By the inductive hypothesis, $\tilde{\mathcal{F}}_{(\eta, \tilde{\xi})} \tilde{\otimes}_{\mathcal{O}_{Y, \eta}} \mathcal{O}_{Z, \eta}$ is $\mathcal{O}_{Z, \eta}$-flat for every $(\eta, \tilde{\xi}) \in|Z| \times U^{\prime}$. To complete the proof, observe that for any $(\eta, \xi) \in|Z| \times U$ with $g(\eta, \xi)=0,(\mathcal{F} / \operatorname{im} \psi)_{(\eta, \xi)}$ is a direct summand of $\tilde{\mathcal{F}}_{(\eta, \tilde{\xi})}$. Indeed, one can show this by a direct calculation based on 'collecting into' the remainder of Weierstrass Division by $g(\eta, \tilde{\xi}, \cdot)$ the remainders of division by the factors of $g(\eta, \tilde{\xi}, \cdot)$. Hence $(\mathcal{F} / \operatorname{im} \psi)_{(\eta, \xi)} \tilde{\otimes}_{\mathcal{O}_{Y, \eta}} \mathcal{O}_{Z, \eta}$ is $\mathcal{O}_{Z, \eta}$-flat, as a direct summand of $\tilde{\mathcal{F}}_{(\eta, \tilde{\xi})} \tilde{\otimes}_{\mathcal{O}_{Y, \eta}} \mathcal{O}_{Z, \eta}$, by [3. Ch. $1, \S 2.3$, Prop. 2].

Remark 2.5 (Frisch's generic flatness theorem [5]). Let $\varphi: X \rightarrow Y$ denote a morphism of complex-analytic spaces and let $\mathcal{F}$ denote a coherent sheaf of $\mathcal{O}_{X}$-modules. Frisch's generic flatness theorem asserts that the non-flat locus $\Sigma:=\{\xi \in X$ : $\mathcal{F}_{\xi}$ is not $\mathcal{O}_{Y, \varphi(\xi)}$-flat $\}$ is a closed analytic subset of $X$ and that if $X$ is reduced, then $\varphi(\Sigma)$ is nowhere dense in $Y$. The first assertion follows from Theorem 2.3 above, together with the fact that $\Sigma$ is a constructible subset of $X$. See [2, Thm. 7.15] for a constructive elementary proof of the latter. The second assertion then follows in a simple way (as in [5, Prop. IV.14]) and, in fact, can also be proved using Theorem 1.1 and further development of (2.1).

\section{Proof of the MAIN THEOREM}

We use the notation preceding Theorem 1.1. Consider a presentation (1.1) of $F$ as an $A$-module. Applying $\tilde{\otimes}_{R} R / \mathfrak{m}$, we get a homomorphism $\Phi_{\mathfrak{m}}: A(0)^{p} \rightarrow A(0)^{q}$ of $A(0)$-modules such that $F \tilde{\otimes}_{R} R / \mathfrak{m} \cong \operatorname{coker}\left(\Phi_{\mathfrak{m}}\right)$. Set $r_{\mathfrak{m}}:=\operatorname{rank}\left(\Phi_{\mathfrak{m}}\right)$. We can assume that $\Phi$ is given by a block matrix (1.4) and $g:=\operatorname{det} \alpha$ satisfies $g(0, x) \neq 0$. For an ideal $J$ in $R$, define

$$
\operatorname{ker}_{J} \Phi:=\left\{\zeta \in A^{p}: \Phi(\zeta) \in J \cdot A^{q}\right\}
$$

and

$\operatorname{rank}_{J} \Phi:=\min \{r \geq 1:$ all $(r+1) \times(r+1)$ minors of $\Phi$ belong to $J \cdot A\}$. 
Our proof of Theorem 1.1(B) is based on showing that property (1) of the theorem is equivalent to equalities $q-l=\operatorname{rank}_{J} \Phi=\operatorname{rank} \Phi_{\mathfrak{m}}$ and that property (2) of the theorem is equivalent to $R / J$-flatness of $\mathcal{G} \tilde{\otimes}_{R} R / J$, where

$$
\mathcal{G}:=A^{r_{\mathrm{m}}} /\left[g \cdot A^{r_{\mathrm{m}}}+\operatorname{im}\left(\alpha^{\#} \cdot \beta\right)\right] .
$$

The latter equivalence is obvious if $g$ is a unit in $A$, since both $F / \operatorname{im} \psi$ and $\mathcal{G}$ are zero in this case. Suppose then that $g$ is not invertible in $A$, that is, $g(0,0)=0$. Since $g(0, x) \neq 0$, then after a (generic and linear) change of the $x$-coordinates to $\left(\tilde{x}, x_{m}\right)$, where $\tilde{x}=\left(x_{1}, \ldots, x_{m-1}\right)$, we have $g\left(0,0, x_{m}\right) \neq 0$. By the Weierstrass Preparation Theorem, $g=u \cdot P$, where $u(0,0) \neq 0$ and $P(y, x)=x_{m}^{d}+\sum_{i=1}^{d} p_{i}(y, \tilde{x}) \cdot x_{m}^{d-i}$, with $p_{i}(0,0)=0$.

The ring $A / g \cdot A$ is a finite free $R\{\tilde{x}\}$-module. We shall describe the action of $\alpha^{\#} \cdot \beta: A^{p-r_{\mathrm{m}}} \rightarrow A^{r_{\mathrm{m}}}$ modulo $g$ as a linear mapping of finite $R\{\tilde{x}\}$-modules. Given $\eta \in A^{p-r_{\mathrm{m}}}$, Weierstrass division by $g$ gives $\eta \equiv \sum_{j=1}^{d} \eta_{j} x_{m}^{d-j}(\bmod g)$, with $\eta_{j} \in R\{\tilde{x}\}^{p-r_{\mathfrak{m}}}$. Applying Weierstrass division by $g$ to the entries of $\alpha^{\#} \cdot \beta$, we form matrices $T_{i}=T_{i}(y, \tilde{x}), 1 \leq i \leq d$, such that

$$
\left(\alpha^{\#} \cdot \beta\right)(\eta) \equiv\left(\sum_{i=1}^{d} T_{i} \cdot x_{m}^{d-i}\right) \cdot\left(\sum_{j=1}^{d} \eta_{j} \cdot x_{m}^{d-j}\right) \quad(\bmod g) .
$$

Applying Euclid division by $P(y, x)$ (as a monic polynomial in $x_{m}$ ) to the latter product, we obtain the matrix $G=\left(G_{i j}\right)_{1 \leq i, j \leq d}$, with block-matrices $G_{i j}$ of size $r_{\mathfrak{m}} \times\left(p-r_{\mathfrak{m}}\right)$ and entries in $R\{\tilde{x}\}$, such that all entries of the matrix

$$
\left(\sum_{i=1}^{d} T_{i} \cdot x_{m}^{d-i}\right) \cdot\left(\sum_{j=1}^{d} \eta_{j} \cdot x_{m}^{d-j}\right)-\sum_{1 \leq i, j \leq d} G_{i j} \cdot \eta_{j} \cdot x_{m}^{d-i}
$$

are linear in the $\eta_{j}$ with coefficients in the ideal generated by $P(y, x)$ in the ring $R\{\tilde{x}\}\left[x_{m}\right]$. Then $\mathcal{G}$ coincides with $R\{\tilde{x}\}^{r_{\mathfrak{m}}} d / \mathrm{im} G$ as $R\{\tilde{x}\}$-modules. With these preparations and modulo Lemma 3.2 below, Theorem 1.1(B) is a consequence of the following.

Proposition 3.1. Let $G: R\{\tilde{x}\}^{\left(p-r_{\mathfrak{m}}\right) d} \rightarrow R\{\tilde{x}\}^{r_{\mathfrak{m}} d}$ be as above (or $G=0$ if $g(0,0) \neq 0)$. Then $\operatorname{ker}\left(\Phi_{\mathfrak{m}}\right)=\left(\operatorname{ker}_{J} \Phi\right)(0)$ if and only if $\operatorname{rank} \Phi_{\mathfrak{m}}=\operatorname{rank}{ }_{J} \Phi$ and $\operatorname{ker}\left(G_{\mathfrak{m}}\right)=\left(\operatorname{ker}_{J} G\right)(0)$.

Before proving Proposition 3.1, let us note that the first equality of Proposition 3.1 expresses $R / J$-flatness of $F$ :

Lemma 3.2. $F \tilde{\otimes}_{R} R / J$ is $R / J$-flat if and only if $\left(\operatorname{ker}_{J} \Phi\right)(0)=\operatorname{ker}\left(\Phi_{\mathfrak{m}}\right)$.

Proof. By definition of $\operatorname{ker}_{J} \Phi, \zeta \in \operatorname{ker}_{J} \Phi$ implies $\Phi(\zeta) \in \mathfrak{m} \cdot A^{q}$, and hence $\Phi_{\mathfrak{m}}(\zeta(0))=0$. Therefore, we always have $\left(\operatorname{ker}_{J} \Phi\right)(0) \subset \operatorname{ker} \Phi_{\mathfrak{m}}$. On the other hand, by a well-known criterion for flatness (see, e.g., [7, Prop. 6.2]), $F \tilde{\otimes}_{R} R / J$ is $R / J$-flat if and only if $\widetilde{\operatorname{Tor}}_{1}^{R / J}\left(F \tilde{\otimes}_{R} R / J, R / \mathfrak{m}\right)=0$.

By (1.2), we have $F \tilde{\otimes}_{R} R / J \cong\left(A^{q} / J \cdot A^{q}\right) / \Phi_{J}\left(A^{p} / J \cdot A^{p}\right)$. Notice that $\operatorname{ker}\left(\Phi_{J}\right)=$ $\left(\operatorname{ker}_{J} \Phi\right) / J \cdot A^{p}$. Hence $\Phi_{J}\left(A^{p} / J \cdot A^{p}\right) \cong\left(A^{p} / J \cdot A^{p}\right) / \operatorname{ker}\left(\Phi_{J}\right) \cong A^{p} / \operatorname{ker}_{J} \Phi$, and we get from (1.2) a short exact sequence

$$
0 \rightarrow A^{p} / \operatorname{ker}_{J} \Phi \rightarrow A^{q} / J \cdot A^{q} \rightarrow F \tilde{\otimes}_{R} R / J \rightarrow 0 .
$$


The induced long exact sequence of $\widetilde{T o r}^{R / J}$-modules ends with

$$
\begin{aligned}
& 0 \rightarrow \widetilde{\operatorname{Tor}}_{1}^{R / J}\left(F \tilde{\otimes}_{R} R / J, R / \mathfrak{m}\right) \rightarrow\left(A^{p} / \operatorname{ker}_{J} \Phi\right) \tilde{\otimes}_{R / J} R / \mathfrak{m} \\
& \stackrel{\lambda}{\rightarrow}\left(A^{q} \tilde{\otimes}_{R} R / J\right) \tilde{\otimes}_{R / J} R / \mathfrak{m} \rightarrow\left(F \tilde{\otimes}_{R} R / J\right) \tilde{\otimes}_{R / J} R / \mathfrak{m} \rightarrow 0,
\end{aligned}
$$

where the leftmost term is zero by $R / J$-flatness of $A^{q} \tilde{\otimes}_{R} R / J$ (which follows from the $R$-flatness of $A^{q}$ ). Thus $F \tilde{\otimes}_{R} R / J$ is $R / J$-flat if and only if

$$
A(0)^{p} /\left(\operatorname{ker}_{J} \Phi\right)(0) \cong\left(A^{p} / \operatorname{ker}_{J} \Phi\right) \tilde{\otimes}_{R / J} R / \mathfrak{m} \stackrel{\lambda}{\longrightarrow} A^{q} \tilde{\otimes}_{R / J} R / \mathfrak{m} \cong A(0)^{q}
$$

is injective. By (1.3), the latter condition is equivalent to $\left(\operatorname{ker}_{J} \Phi\right)(0) \supset \operatorname{ker}\left(\Phi_{\mathfrak{m}}\right)$, which completes the proof of the lemma.

The proof of Proposition 3.1 depends on several lemmas, which follow. First, we establish a useful cancellation law.

Lemma 3.3. Let $J$ be an ideal in $R$, and let $g, \zeta \in A$ be such that $g(0, x) \neq 0$ in $A(0)=\mathbb{K}\{x\}$ and $g \cdot \zeta \in J \cdot A$. Then $\zeta \in J \cdot A$.

Proof. Write $\zeta=\sum_{\nu \in \mathbb{N} m} \zeta_{\nu} x^{\nu}$, where $\zeta_{\nu} \in R$, and consider $g$ and $\zeta$ as elements of the ring $\tilde{A}:=R[[x]]$. By assumption, $g \notin \mathfrak{m} \cdot \tilde{A}$. Hence, after localizing in $\mathfrak{m} \cdot \tilde{A}$, we get $\zeta_{\mathfrak{m} \tilde{A}} \in(J \tilde{A})_{\mathfrak{m} \tilde{A}}$, because $g_{\mathfrak{m} \tilde{A}}$ is invertible in $\tilde{A}_{\mathfrak{m} \tilde{A}}$. Since $\tilde{A}$ is a free $R$-module, we have $\tilde{A}_{\mathfrak{m} \tilde{A}} \cong R_{\mathfrak{m}}[[x]]$, and hence $\zeta_{\mathfrak{m} \tilde{A}} \in(J \tilde{A})_{\mathfrak{m} \tilde{A}}$ if and only if, for all $\nu \in \mathbb{N}^{m}$, $\left(\zeta_{\nu}\right)_{\mathfrak{m}} \in J_{\mathfrak{m}}$, that is, $\zeta_{\nu} \in J$. Thus $\zeta \in J \cdot A$, as required.

Recall that $r_{\mathfrak{m}}$ denotes the rank of $\Phi_{\mathfrak{m}}$ (in the notation at the beginning of this section).

Lemma 3.4. Let $J$ be an ideal in $R$. Then the following conditions are equivalent:

(i) $\operatorname{rank} \Phi_{\mathfrak{m}}=\operatorname{rank}_{J} \Phi$;

(ii) we can order the columns and rows of $\Phi$ so that $\Phi$ has block form (1.4) with $\alpha$ of size $r \times r,(\operatorname{det} \alpha)(0, x) \neq 0$ and $\operatorname{rank}_{J} \Phi=r$;

(iii) we can order the columns and rows of $\Phi$ so that $\Phi$ has block form (1.4), where $\alpha$ has size $r \times r,(\operatorname{det} \alpha)(0, x) \neq 0$, and all entries of $(\operatorname{det} \alpha) \cdot \delta-\gamma \cdot \alpha^{\#} \cdot \beta$ are in $J \cdot A$;

(iv) if $\Phi$ is a block matrix (1.4), where $\alpha$ is of size $r_{\mathfrak{m}} \times r_{\mathfrak{m}}$ and $(\operatorname{det} \alpha)(0) \neq 0$, then all entries of $(\operatorname{det} \alpha) \cdot \delta-\gamma \cdot \alpha^{\#} \cdot \beta$ are in $J \cdot A$;

(v) if $g \in A, g(0, x) \neq 0$, and $A^{q}=A^{r} \oplus A^{l}$, where $g \cdot A^{q} \subset \operatorname{im} \Phi+\left(\{0\}^{r} \oplus A^{l}\right)$ and $\operatorname{im} \Phi \cap\left(\{0\}^{r} \oplus A^{l}\right) \subset\{0\}^{r} \oplus \mathfrak{m} \cdot A^{l}$, then $\operatorname{im} \Phi \cap\left(\{0\}^{r} \oplus A^{l}\right) \subset\{0\}^{r} \oplus J \cdot A^{l}$;

(vi) if $g \in A, g(0, x) \neq 0$ and $\psi: A^{l} \rightarrow F$ is a homomorphism of A-modules such that $g \cdot F \subset \operatorname{im} \psi$ and $\operatorname{ker} \psi \subset \mathfrak{m} \cdot A^{l}$, then $\operatorname{ker} \psi \subset J \cdot A^{l}$.

Proof. (ii) $\Rightarrow$ (i): Clearly $r \leq \operatorname{rank} \Phi_{\mathfrak{m}}$ and $\operatorname{rank} \Phi_{\mathfrak{m}} \leq \operatorname{rank}_{J} \Phi$. Hence all three are equal if $\operatorname{rank}_{J} \Phi=r$.

(i) $\Rightarrow$ (iv): By Remark 1.3, all entries of $(\operatorname{det} \alpha) \cdot \delta-\gamma \cdot \alpha^{\#} \cdot \beta$ are $\left(r_{\mathfrak{m}}+1\right) \times\left(r_{\mathfrak{m}}+1\right)$ minors of $\Phi$, and hence they belong to $J \cdot A$ if $\operatorname{rank}_{J} \Phi=r_{\mathfrak{m}}$.

(iv) $\Rightarrow$ (iii): Set $r=r_{\mathfrak{m}}$ and let $\alpha, \beta, \gamma, \delta$ be as in (iv).

(iii) $\Rightarrow$ (ii): Set $g=\operatorname{det} \alpha$. By the matrix identity of Remark 1.3, all $(r+1) \times$ $(r+1)$ minors of $g \cdot \Phi$ are combinations of the entries of $(\operatorname{det} \alpha) \cdot \delta-\gamma \cdot \alpha^{\#} \cdot \beta$ with coefficients in $A$. Hence, if $\zeta$ is an $(r+1) \times(r+1)$ minor of $\Phi$, then $g^{r+1} \cdot \zeta \in J \cdot A$, which by Lemma 3.3 implies $\zeta \in J \cdot A$. 
$(\mathrm{v}) \Rightarrow(\mathrm{vi}):$ The homomorphism $\psi: A^{l} \rightarrow F$ can be extended to a surjective homomorphism $\Psi: A^{q} \rightarrow F$, which by Oka's coherence theorem extends to an exact sequence $A^{p} \stackrel{\Phi}{\longrightarrow} A^{q} \stackrel{\Psi}{\longrightarrow} F \rightarrow 0$.

$(\mathrm{vi}) \Rightarrow(\mathrm{v})$ : The assumptions in (v) imply the assumptions in (vi), with the same $g$ and $\psi$ being the restriction of $\Psi$ (from the above exact sequence) to $\{0\}^{r} \oplus A^{l}$. Then $\operatorname{im} \Phi \cap\left(\{0\}^{r} \oplus A^{l}\right)=\operatorname{ker} \psi \subset J \cdot A^{l}$.

It remains to show that (iv) is equivalent to (v). Write $\Phi$ in block form (1.4), with $\alpha$ of size $r \times r$. We will use the fact that $(\varrho, \sigma) \in A^{q}=A^{r} \oplus A^{l}$ belongs to $\operatorname{im} \Phi \cap\left(\{0\}^{r} \oplus A^{l}\right)$ if and only if $\sigma=\gamma \xi+\delta \eta$ and $\alpha \xi+\beta \eta=\varrho=0$, for some $(\xi, \eta) \in A^{r} \oplus A^{p-r}$. Then $(\operatorname{det} \alpha) \cdot \xi=\left(\alpha^{\#} \cdot \alpha\right)(\xi)=-\left(\alpha^{\#} \cdot \beta\right)(\eta)$, and hence

$$
(\operatorname{det} \alpha) \cdot \sigma=\gamma((\operatorname{det} \alpha) \cdot \xi)+(\operatorname{det} \alpha) \cdot \delta(\eta)=-\left(\gamma \cdot \alpha^{\#} \cdot \beta\right)(\eta)+(\operatorname{det} \alpha) \cdot \delta(\eta)
$$

It follows that

$(\operatorname{det} \alpha) \cdot\left[\operatorname{im} \Phi \cap\left(\{0\}^{r} \oplus A^{l}\right)\right] \subset\{0\}^{r} \oplus \operatorname{im}\left[(\operatorname{det} \alpha) \cdot \delta-\gamma \cdot \alpha^{\#} \cdot \beta\right] \subset \operatorname{im} \Phi \cap\left(\{0\}^{r} \oplus A^{l}\right)$,

where the latter inclusion is a consequence of Remark 1.3.

$(\mathrm{v}) \Rightarrow(\mathrm{iv})$ : The assumptions of (iv) imply that all entries of $(\operatorname{det} \alpha) \cdot \delta-\gamma \cdot \alpha^{\#} \cdot \beta$ are in $\mathfrak{m} \cdot A$ (by Remark 1.3, as $\left(r_{\mathfrak{m}}+1\right) \times\left(r_{\mathfrak{m}}+1\right)$ minors of $\left.\Phi\right)$. Therefore the assumptions of (v) follow with $r:=r_{\mathfrak{m}}, l:=q-r$ and $g:=\operatorname{det} \alpha$. Indeed, $g \cdot \operatorname{Id}_{r}=\alpha \cdot \alpha^{\#}$, and so

$$
g \cdot A^{q} \subset \alpha\left(A^{r}\right) \oplus A^{l} \subset \operatorname{im} \Phi+\left(\{0\}^{r} \oplus A^{l}\right) .
$$

Also, by (3.3),$\zeta=(\varrho, \sigma) \in \operatorname{im} \Phi \cap\left(\{0\}^{r} \oplus A^{l}\right)$ implies $g \cdot \sigma \in \operatorname{im}\left[(\operatorname{det} \alpha) \cdot \delta-\gamma \cdot \alpha^{\#} \cdot \beta\right]$. Hence $g \cdot \zeta \in \mathfrak{m} \cdot A^{q}$, and therefore $\zeta \in \mathfrak{m} \cdot A^{q}$, by Lemma 3.3 .

Now, (v) implies $\operatorname{im} \Phi \cap\left(\{0\}^{r} \oplus A^{l}\right) \subset\{0\}^{r} \oplus J \cdot A^{l}$, which by (3.3) means that $\operatorname{im}\left[(\operatorname{det} \alpha) \cdot \delta-\gamma \cdot \alpha^{\#} \cdot \beta\right] \subset J \cdot A^{l}$, and hence the entries of $(\operatorname{det} \alpha) \cdot \delta-\gamma \cdot \alpha^{\#} \cdot \beta$ are in $J \cdot A$.

(iv) $\Rightarrow(\mathrm{v})$ : Let $\pi_{1}: A^{q}=A^{r} \oplus A^{l} \rightarrow A^{r}$ denote the canonical projection to the first direct summand. By the assumptions of $(\mathrm{v})$, there is a matrix $\Xi$ of size $p \times r$ with entries in $A$ such that $g \cdot \operatorname{Id}_{r}=\pi_{1} \cdot \Phi \cdot \Xi$. Since $g(0, x) \neq 0$, it follows that rank $\left(\pi_{1} \cdot \Phi\right)=r$. Therefore there is an ordering of columns of $\Phi$ such that $\pi_{1} \cdot \Phi=[\alpha, \beta]$, with $\alpha$ of size $r \times r$ and $(\operatorname{det} \alpha)(0, x) \neq 0$. Then $\Phi$ has block form (1.4) and $\{0\}^{r} \oplus \operatorname{im}\left[(\operatorname{det} \alpha) \cdot \delta-\gamma \cdot \alpha^{\#} \cdot \beta\right] \subset \operatorname{im} \Phi \cap\left(\{0\}^{r} \oplus A^{l}\right)$. Hence, by the assumptions of $(\mathrm{v})$, all entries of $(\operatorname{det} \alpha) \cdot \delta-\gamma \cdot \alpha^{\#} \cdot \beta$ are in $\mathfrak{m} \cdot A^{l}$. Using the equivalence of (ii) and (iii) for $J=\mathfrak{m}$, we see that $r=\operatorname{rank}_{\mathfrak{m}} \Phi=\operatorname{rank} \Phi_{\mathfrak{m}}$; i.e., the assumptions of (iv) are satisfied. It follows that $J \cdot A^{l} \supset \operatorname{im}\left[(\operatorname{det} \alpha) \cdot \delta-\gamma \cdot \alpha^{\#} \cdot \beta\right]$; hence $\{0\}^{r} \oplus J \cdot A^{l} \supset(\operatorname{det} \alpha) \cdot\left[\operatorname{im} \Phi \cap\left(\{0\}^{r} \oplus A^{l}\right)\right]$, by (3.3), and thus $\{0\}^{r} \oplus J \cdot A^{l} \supset$ $\operatorname{im} \Phi \cap\left(\{0\}^{r} \oplus A^{l}\right)$, by Lemma 3.3 .

Lemma 3.5. Assume that $\operatorname{ker}\left(\Phi_{\mathfrak{m}}\right)=\left(\operatorname{ker}_{J} \Phi\right)(0)$. Then $\operatorname{rank} \Phi_{\mathfrak{m}}=\operatorname{rank}_{J} \Phi$.

Proof. Clearly, $r_{\mathfrak{m}}=\operatorname{rank} \Phi_{\mathfrak{m}} \leq \operatorname{rank}_{J} \Phi$. For the opposite inequality, choose $\xi_{j}(x) \in \operatorname{ker} \Phi_{\mathfrak{m}} \subset \mathbb{K}\{x\}^{p}, 1 \leq j \leq p-r_{\mathfrak{m}}$, so that the $p \times\left(p-r_{\mathfrak{m}}\right)$ matrix $\xi(x)=\left[\xi_{1}(x), \ldots, \xi_{p-r_{\mathfrak{m}}}(x)\right]$ has rank $p-r_{\mathfrak{m}}$. Then, by assumption, there is a matrix $\Xi=\Xi(y, x)$ of size $p \times\left(p-r_{\mathfrak{m}}\right)$ such that the entries of $\Phi \cdot \Xi$ are in $J \cdot A$ and $\Xi(0, x)=\xi(x)$. It follows that rank $\Xi=p-r_{\mathfrak{m}}$. By Cramer's Rule (and after an 
appropriate reordering of the columns of $\Phi$ and rows of $\Xi$ ), there exists a matrix $\Sigma$ of size $\left(p-r_{\mathfrak{m}}\right) \times\left(p-r_{\mathfrak{m}}\right)$ with entries in $A$ such that

$$
\Xi \cdot \Sigma=\left[\begin{array}{c}
g \cdot \operatorname{Id}_{p-r_{\mathfrak{m}}} \\
\Gamma
\end{array}\right],
$$

where $g \in A$ satisfies $g(0, x) \neq 0$ and $\Gamma$ is a matrix with entries in $A$ of size $r_{\mathfrak{m}} \times\left(p-r_{\mathfrak{m}}\right)$. Write $\Phi=\left[\Phi_{1}, \Phi_{2}\right]$, where $\Phi_{1}$ consists of the first $p-r_{\mathfrak{m}}$ columns of $\Phi$. It follows that $g \cdot \Phi_{1}+\Phi_{2} \cdot \Gamma$ is a matrix with entries in $J \cdot A$, and hence the entries of $g \cdot \Phi-\left[-\Phi_{2} \cdot \Gamma, g \cdot \Phi_{2}\right]$ are also in $J \cdot A$. Since $\Phi_{2}$ is of size $q \times r_{\mathfrak{m}}$, then $\operatorname{rank}\left[-\Phi_{2} \cdot \Gamma, g \cdot \Phi_{2}\right] \leq \operatorname{rank} \Phi_{2} \leq r_{\mathfrak{m}}$. Consequently,

$$
\operatorname{rank}_{J}(g \cdot \Phi)=\operatorname{rank}_{J}\left[-\Phi_{2} \cdot \Gamma, g \cdot \Phi_{2}\right] \leq r_{\mathfrak{m}} .
$$

It thus suffices to show that $\operatorname{rank}_{J} \Phi=\operatorname{rank}_{J}(g \cdot \Phi)$, but that is a consequence of Lemma 3.3

Remark 3.6. Let $\Phi$ be as in Lemma 3.4(iv), and let $\pi_{2}: A^{p}=A^{r_{\mathfrak{m}}} \oplus A^{p-r_{\mathfrak{m}}} \rightarrow$ $A^{p-r_{\mathrm{m}}}$ denote the canonical projection to the second direct summand. Then

$$
\left(\operatorname{ker}_{J} \Phi\right)(0)=\operatorname{ker}\left(\Phi_{\mathfrak{m}}\right) \text { iff } \pi_{2}\left(\left(\operatorname{ker}_{J} \Phi\right)(0)\right)=\pi_{2}\left(\operatorname{ker}\left(\Phi_{\mathfrak{m}}\right)\right)
$$

where $J$ is an ideal in $R$. Indeed, since $\left(\operatorname{ker}_{J} \Phi\right)(0)$ is always contained in $\operatorname{ker}\left(\Phi_{\mathfrak{m}}\right)$ (cf. the proof of Lemma 3.2), it suffices to show that $\pi_{2}\left(\left(\operatorname{ker}_{J} \Phi\right)(0)\right) \supset \pi_{2}\left(\operatorname{ker}\left(\Phi_{\mathfrak{m}}\right)\right)$ implies $\operatorname{ker}\left(\Phi_{\mathfrak{m}}\right) \subset\left(\operatorname{ker}_{J} \Phi\right)(0)$. Let $\zeta=\zeta(x)$ be an element of $\operatorname{ker} \Phi_{\mathfrak{m}}$, and let $\xi \in \operatorname{ker}_{J} \Phi$ be such that $\pi_{2}(\xi(0, x))=\pi_{2}(\zeta)$. It suffices to show that $\zeta(x)=\xi(0, x)$. Since $\eta(x):=\xi(0, x)-\zeta(x)$ belongs to $\operatorname{ker} \pi_{2} \cap \operatorname{ker} \Phi_{\mathfrak{m}}$, it follows that $\eta=\left(\eta^{\prime}, 0\right) \in$ $A^{r_{\mathfrak{m}}} \oplus A^{p-r_{\mathfrak{m}}}$ and $\alpha(0, x) \cdot \eta^{\prime}(x)=0$. Therefore $(\operatorname{det} \alpha)(0, x) \cdot \eta^{\prime}(x)=0$, and hence $\eta^{\prime}=0$, and $\eta=0$, as required.

Lemma 3.7. Let $\Phi$ and $\pi_{2}: A^{p}=A^{r_{\mathfrak{m}}} \oplus A^{p-r_{\mathfrak{m}}} \rightarrow A^{p-r_{\mathfrak{m}}}$ be as above, and let $J$ be an ideal in $R$. Then $\eta \in \pi_{2}\left(\operatorname{ker}_{J} \Phi\right)$ if and only if the following two conditions hold:

$$
\begin{gathered}
\left(\alpha^{\#} \cdot \beta\right)(\eta) \in g \cdot A^{r_{\mathfrak{m}}}+J \cdot A^{r_{\mathfrak{m}}}, \\
\left(g \cdot \delta-\gamma \cdot \alpha^{\#} \cdot \beta\right)(\eta) \in J \cdot A^{q-r_{\mathfrak{m}}},
\end{gathered}
$$

where $g$ denotes $\operatorname{det} \alpha$.

Proof. For the "only if" direction, let $(\xi, \eta)$ be an element of $\operatorname{ker}_{J} \Phi$. Then $\alpha \xi+\beta \eta \in$ $J \cdot A^{r_{\mathfrak{m}}}$ and $\gamma \xi+\delta \eta \in J \cdot A^{q-r_{\mathfrak{m}}}$, and hence

$$
\begin{gathered}
g \cdot \xi+\left(\alpha^{\#} \cdot \beta\right)(\eta)=\alpha^{\#} \cdot(\alpha \xi+\beta \eta) \equiv 0\left(\bmod J \cdot A^{r_{\mathfrak{m}}}\right) \text { and } \\
\left(g \cdot \delta-\gamma \cdot \alpha^{\#} \cdot \beta\right)(\eta)=g \cdot(\gamma \xi+\delta \eta)-\gamma \cdot\left(g \cdot \xi+\left(\alpha^{\#} \cdot \beta\right)(\eta)\right) \equiv 0\left(\bmod J \cdot A^{q-r_{\mathfrak{m}}}\right) .
\end{gathered}
$$

Now, for the "if" direction, let $\xi \in A^{r_{\mathfrak{m}}}$ be such that $g \cdot \xi+\left(\alpha^{\#} \cdot \beta\right)(\eta) \equiv 0$ modulo $J \cdot A^{r_{\mathfrak{m}}}$ and assume that $\left(g \cdot \delta-\gamma \cdot \alpha^{\#} \cdot \beta\right)(\eta) \in J \cdot A^{q-r_{\mathfrak{m}}}$. Then

$$
\begin{gathered}
g \cdot(\alpha \xi+\beta \eta)=\alpha \cdot\left(g \cdot \xi+\left(\alpha^{\#} \cdot \beta\right)(\eta)\right) \equiv 0\left(\bmod J \cdot A^{r_{\mathfrak{m}}}\right) \quad \text { and } \\
g \cdot(\gamma \xi+\delta \eta)=\left(g \cdot \delta-\gamma \cdot \alpha^{\#} \cdot \beta\right)(\eta)+\gamma \cdot\left(g \cdot \xi+\left(\alpha^{\#} \cdot \beta\right)(\eta)\right) \equiv 0\left(\bmod J \cdot A^{q-r_{\mathfrak{m}}}\right) .
\end{gathered}
$$

Therefore $g \cdot(\xi, \eta) \in \operatorname{ker}_{J} \Phi ;$ hence $(\xi, \eta) \in \operatorname{ker}_{J} \Phi$ by Lemma 13.3 , and so $\eta \in$ $\pi_{2}\left(\operatorname{ker}_{J} \Phi\right)$, as required. 
Remark 3.8. Since the entries of $g \cdot \delta-\gamma \cdot \alpha^{\#} \cdot \beta$ are in $\mathfrak{m} \cdot A$ (by Remark 1.3), Lemma 3.7 applied to $J=\mathfrak{m}$ asserts that

$$
\eta \in \pi_{2}\left(\operatorname{ker}\left(\Phi_{\mathfrak{m}}\right)\right) \quad \text { iff } \quad\left(\alpha^{\#} \cdot \beta\right)(0, x) \cdot \eta(x) \in g(0, x) \cdot A(0)^{r_{\mathfrak{m}}} .
$$

Proof of Proposition 3.1. Let $\pi_{2}: A^{p}=A^{r_{\mathfrak{m}}} \oplus A^{p-r_{\mathfrak{m}}} \rightarrow A^{p-r_{\mathfrak{m}}}$ be as above. By Lemma 3.5 and Remark [3.6, it suffices to show the equivalence

$$
\pi_{2}\left(\left(\operatorname{ker}_{J} \Phi\right)(0)\right)=\pi_{2}\left(\operatorname{ker}\left(\Phi_{\mathfrak{m}}\right)\right) \quad \text { iff } \quad\left(\operatorname{ker}_{J} G\right)(0)=\operatorname{ker}\left(G_{\mathfrak{m}}\right),
$$

under the assumption that $r_{\mathfrak{m}}=\operatorname{rank}_{J} \Phi$ (i.e., the equivalent conditions of Lemma 3.4 are satisfied).

Suppose first that $g$ is a unit in $A$ (and hence $\left(\operatorname{ker}_{J} G\right)(0)=\operatorname{ker}\left(G_{\mathfrak{m}}\right)$ trivially). Then the condition $\left(\alpha^{\#} \cdot \beta\right)(\eta) \in g \cdot A^{r_{\mathfrak{m}}}+J \cdot A^{r_{\mathfrak{m}}}$ of Lemma 3.7 is vacuous, because $g \cdot A^{r_{\mathrm{m}}}+J \cdot A^{r_{\mathrm{m}}}=A^{r_{\mathrm{m}}}$. Since the entries of $g \cdot \delta-\gamma \cdot \alpha^{\#} \cdot \beta$ are in $J \cdot A$ (Lemma 3.4(iv)), it follows from Lemma 3.7 that $\pi_{2}\left(\operatorname{ker}_{J} \Phi\right)=A^{p-r_{\mathfrak{m}}}$. Therefore $\pi_{2}\left(\left(\operatorname{ker}_{J} \Phi\right)(0)\right) \supset \pi_{2}\left(\operatorname{ker}\left(\Phi_{\mathfrak{m}}\right)\right)$, and hence $\left(\operatorname{ker}_{J} \Phi\right)(0)=\operatorname{ker}\left(\Phi_{\mathfrak{m}}\right)$, by Remark 3.6.

Suppose then that $g$ is not a unit in $A$, i.e., $g(0,0)=0$. Let $\eta=\eta(x) \in A(0)^{p-r_{\mathrm{m}}}$. Since $g(0, x) \neq 0$, then after a generic linear change of the $x$-variables, $g$ is regular in $x_{m}$. Applying the Weierstrass division theorem, we get

$$
\eta(x)=\sum_{j=1}^{d} \eta_{j}(\tilde{x}) \cdot x_{m}^{d-j}+g(0, x) \cdot \tilde{q}(x),
$$

where $\tilde{x}=\left(x_{1}, \ldots, x_{m}\right)$. Hence, by Remark 3.8 ,

$$
\eta \in \pi_{2}\left(\operatorname{ker}\left(\Phi_{\mathfrak{m}}\right)\right) \quad \text { iff } \quad\left(\alpha^{\#} \cdot \beta\right)(0, x) \cdot\left(\sum_{j=1}^{d} \eta_{j}(\tilde{x}) \cdot x_{m}^{d-j}\right) \in g(0, x) \cdot A(0)^{r_{\mathfrak{m}}} .
$$

By (3.1) and (3.2), the latter is the case if and only if $\left\{\eta_{j}(\tilde{x})\right\}_{j=1}^{d} \in \operatorname{ker}\left(G_{\mathfrak{m}}\right)$.

Finally, let $\eta=\eta(y, x) \in A^{p-r_{\mathfrak{m}}}$. By the Weierstrass division theorem (after a linear change of the $x$-variables, if needed),

$$
\eta(y, x)=\sum_{j=1}^{d} \eta_{j}(y, \tilde{x}) \cdot x_{m}^{d-j}+g(y, x) \cdot \tilde{q}(y, x),
$$

where $\tilde{x}=\left(x_{1}, \ldots, x_{m}\right)$. Since the entries of $g \cdot \delta-\gamma \cdot \alpha^{\#} \cdot \beta$ are in $J \cdot A$ (Lemma $3.4($ iv)), Lemma 3.7 implies that

$$
\eta \in \pi_{2}\left(\operatorname{ker}_{J} \Phi\right) \quad \text { iff } \quad\left(\alpha^{\#} \cdot \beta\right)\left(\sum_{j=1}^{d} \eta_{j}(y, \tilde{x}) \cdot x_{m}^{d-j}\right) \in g \cdot A^{r_{\mathrm{m}}}+J \cdot A^{r_{\mathrm{m}}} .
$$

By (3.1) and (3.2), the latter is the case if and only if $\left\{\eta_{j}(y, \tilde{x})\right\}_{j=1}^{d} \in \operatorname{ker}_{J} G$, which completes the proof of Proposition 3.1 and Theorem 1.1

\section{REFERENCES}

1. J. Adamus, E. Bierstone and P.D. Milman, Geometric Auslander criterion for flatness, preprint 2009, arXiv:0901.2744v4, to appear in Amer. J. Math.

2. E. Bierstone and P.D. Milman, The local geometry of analytic mappings, Università di Pisa, ETS Editrice, Pisa, Italy, 1988. MR971251 (90j:32011)

3. N. Bourbaki, Commutative Algebra, Elements of Mathematics, Springer, Berlin, 1989. MR2333539 (2008h:13001)

4. A. Douady, Le problème des modules pour les sous-espaces analytiques compacts d'un espace analytique donné, Ann. Inst. Fourier (Grenoble) 16:1 (1966), 1-95. MR0203082 (34:2940) 
5. J. Frisch, Points de platitude d'un morphisme d'espaces analytiques complexes, Invent. Math. 4 (1967), 118-138. MR0222336 (36:5388)

6. A. Galligo and M. Kwieciński, Flatness and fibred powers over smooth varieties, J. Algebra 232 (2000), 48-63. MR.1783912 (2001i:14006)

7. H. Hironaka, Stratification and flatness, Real and Complex Singularities, Proc. Oslo, 1976, ed. Per Holm, Sijthoff and Noordhoff (1977), 199-265. MR0499286 (58:17187)

Department of Mathematics, The University of Western Ontario, London, Ontario, Canada N6A 5B7 - And - Institute of Mathematics, Faculty of Mathematics and Computer Science, Jagiellonian University, ul. Łojasiewicza 6, 30-348 Kraków, Poland

E-mail address: jadamus@uwo.ca

The Fields Institute, 222 College Street, Toronto, Ontario, Canada M5T 3J1 and - Department of Mathematics, University of Toronto, Toronto, Ontario, Canada M5S 2E4

E-mail address: bierston@fields.utoronto.ca

Department of Mathematics, University of Toronto, Toronto, Ontario, Canada M5S 2E4

E-mail address: milman@math.toronto.edu 